\title{
SPECT/CT-Guided Ultrasound for Parathyroid Adenoma Localization: A 1-Stop Approach
}

\author{
Philip Touska ${ }^{1}$, Alison Elstob ${ }^{1}$, Nikhil Rao ${ }^{2}$, and Arum Parthipun ${ }^{3}$ \\ ${ }^{1}$ Department of Radiology, St. George's Hospital, London, United Kingdom; ${ }^{2}$ Department of Radiology, City Hospital Birmingham, \\ Birmingham, United Kingdom; and ${ }^{3}$ Department of Nuclear Medicine, Royal Free London NHS Foundation Trust, London, United \\ Kingdom
}

Our rationale was to evaluate the accuracy of 1-stop (single patient-attendance) SPECT/CT-guided ultrasound in the localization of parathyroid adenomata. Secondary aims included analyzing the effect of multiple parathyroid adenomata and concurrent thyroid disease on sensitivity. Methods: Patients with hyperparathyroidism who had undergone parathyroidectomy were identified over a 5-y period. Pathologic correlation with results from preoperative $99 \mathrm{mT}$ T-sestamibi SPECT/CT followed by targeted ultrasound of the neck was performed. The number of glands, the location, and the presence of concurrent thyroid disease were reviewed. Results: The study included 146 patients (88\% single gland, $7 \%$ multigland, and $5 \%$ negative explorations). The sensitivity and specificity of SPECT/CT-guided ultrasound were $83 \%$ and $96 \%$, respectively. The sensitivity was higher for single gland $(87 \%)$ than multigland disease $(70 \%)$. The addition of ultrasound significantly increased the sensitivity of the technique $(P<0.001)$. The presence of concurrent thyroid disease (nodules/thyroiditis) did not adversely affect sensitivity (85\% confidence interval, $74.2 \%-$ $93.1 \%$ ) compared with normal or atrophic glands (82\% confidence interval, 72.3\%-89.7\%). Conclusion: SPECT/CT-guided ultrasound represents a useful means of localizing parathyroid adenomata, thereby aiding the decision to undertake minimally invasive or exploratory surgery. The 1-stop approach offers patient convenience and enables the radiologist to use the additive benefits of both modalities to optimize localization. The technique is less sensitive in multigland disease, but concurrent thyroid disease does not adversely affect sensitivity.

Key Words: parathyroid adenoma; parathyroidectomy; parathyroid

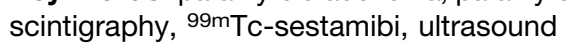

J Nucl Med Technol 2019; 47:64-69

DOI: 10.2967/jnmt.118.209767

$\mathbf{P}$ tered, endocrinologic disorder characterized by excessive secretion of parathyroid hormone and consequent hypercalcemia. The condition results in a protean, but well-established, constellation of clinical features, ranging from renal

\footnotetext{
Received Feb. 15, 2018; revision accepted Aug. 14, 2018.

For correspondence or reprints contact: Arum Parthipun, Department of Nuclear Medicine, Royal Free London NHS Foundation Trust, London, NW3 2QG, U.K.

E-mail: arum.parthipun@nhs.net

Published online Aug. 23, 2018.

COPYRIGHT (c) 2019 by the Society of Nuclear Medicine and Molecular Imaging.
}

tract calcification and pancreatitis to neuropsychiatric disorders and cardiac arrhythmias. It is most commonly caused by parathyroid adenomata or hyperplastic glands, which may be single $(80 \%-85 \%)$ or multiple $(10 \%-15 \%)(1)$. More infrequently, it may occur in the setting of familial syndromes, such as multiple endocrine neoplasia or parathyroid carcinoma. Curative management and restoration of normal calcium homeostasis may be achieved by parathyroidectomy, which involves resection of the affected gland or glands. Historically, the procedure of choice was an open, 4-gland exploration. However, the advent of preoperative localization studies has enabled selective, minimally invasive parathyroidectomy. This technique significantly reduces operative risk and is associated with an improved aesthetic result and reduced recovery time $(2,3)$. Therefore, it has become increasingly important for medical imagers to provide accurate preoperative localization information, which can be used to determine surgical technique.

Variation in parathyroid gland anatomy presents a significant challenge to imaging-based localization; therefore, an appreciation of the relevant embryology is of paramount importance. The superior parathyroid glands, derived from the fourth branchial pouch, have a fairly constant position, typically lying on the posterior surface of the upper thyroid gland and dorsal to the recurrent laryngeal nerve (4), but the positions of the inferior parathyroid glands, derived from the third branchial pouch, are more likely to be variable, occurring along the path of descent of the parathymic complex.

Supernumerary glands occur in approximately $5 \%$ of cases and are typically located in the region of the thymus and thyrothymic ligament (5).

The first role of imaging in primary hyperparathyroidism involves identification of cases of multigland disease, which would obviate a minimally invasive approach and require exploration. Such cases may have existing risk factors, such as multiple endocrine neoplasia or a familial predisposition. The second role of imaging involves anatomic localization, which requires an appreciation of the relevant anatomy and embryology. The third role involves identification of occult thyroid disease, which may alter surgical management (e.g., requiring a concurrent thyroidectomy).

The primary aim was to determine the sensitivity and specificity of a 1-stop approach to parathyroid localization 
using SPECT/CT-guided ultrasound within our institution. Secondary aims included determining whether the techniques were additive and whether sensitivity and specificity were adversely affected by the presence of multiple glands or concurrent thyroid disease.

\section{MATERIALS AND METHODS}

\section{Patient Selection}

The records of patients who had undergone parathyroidectomy for primary hyperparathyroidism between October 2009 and March 2015 were reviewed. This 5.4-y period corresponded with SPECT/CT-guided ultrasound as the standard parathyroid localization technique at our institution. Local pathology databases were utilized to identify the details of patients who had undergone parathyroidectomy for primary hyperparathyroidism. The picture archiving and communication system and radiology information system databases were used to retrieve imaging reports. Pre- and postoperative calcium and parathyroid hormone levels were also reviewed to confirm operative success.

\section{Imaging Technique}

One-stop (single-attendance) preoperative SPECT/CT-guided ultrasound was the standard protocol during the evaluation period. This involved a single operator using the SPECT/CT findings to guide ultrasound scanning and ultimately reviewing both examinations together to reach a diagnosis.

The parathyroid scintigraphy was performed after an intravenous injection of $750 \mathrm{MBq}$ of ${ }^{99 \mathrm{~m}} \mathrm{Tc}$-sestamibi. Imaging was performed using a TruePoint Symbia T6 SPECT/CT scanner (Siemens Healthcare $\mathrm{GmbH}$ ). Planar imaging of the neck and upper mediastinum was performed at 15 and $150 \mathrm{~min}$ after tracer injection. Planar images were acquired with a low-energy highresolution collimator, a $256 \times 256$ matrix, and a 2.29 zoom factor over $10 \mathrm{~min}$. A SPECT/CT acquisition was performed during the delayed phase using $110 \mathrm{kV}, 190 \mathrm{mAs}$, and 3-mm slice thickness. Radionuclide scans were called positive if increased uptake seen on early imaging showed retention on delayed scans relative to the thyroid and localized to a nodule in a location typical for a parathyroid adenoma on CT.

After review of the SPECT/CT images, a targeted ultrasound scan was performed using a $14-\mathrm{MHz}$ high-resolution linear probe on a Logiq E9 ultrasound scanner (GE Healthcare). This involved evaluating the location of abnormal uptake identified on SPECT/ CT on ultrasound to confirm the presence of a parathyroid adenoma and to note its anatomic location; the neck was also subsequently scanned for any additional abnormal glands not visible on SPECT/CT. In the event that no abnormal glands were localized on SPECT/CT, ultrasound was used to scan the neck for adenomata in all possible locations accessible on ultrasound. Typical ultrasound features of a parathyroid adenoma are of a hypoechoic nodule, usually separate from the thyroid, in a typical location. Additional sonographic features include hypervascularity and a separate vascular pedicle.

The SPECT/CT findings were reviewed by a single experienced consultant (attending) radiologist with specialist training in radionuclide imaging, who performed the subsequent ultrasound examinations on all patients.

Figure 1 shows an example of the multimodality approach to parathyroid gland localization.
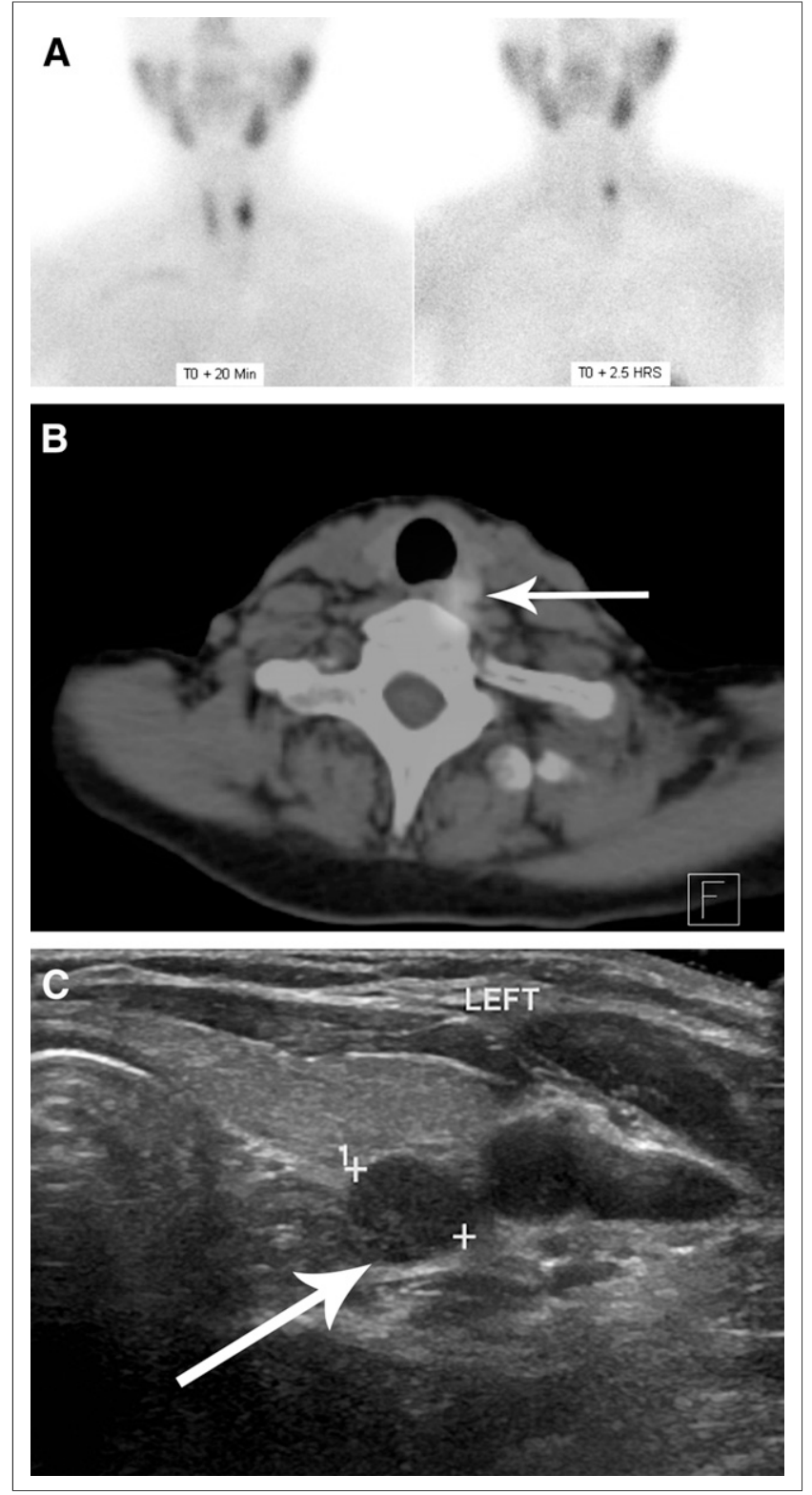

FIGURE 1. Multimodality approach to parathyroid localization. Left superior parathyroid adenoma is diagnosed using both $99 \mathrm{mTC}$-sestamibi SPECT/CT and ultrasound. (A) Planar radionuclide imaging performed after ${ }^{99 \mathrm{~m} T c-s e s t a m i b i}$ injection at $20 \mathrm{~min}$ and $2.5 \mathrm{~h}$ after injection. Planar image demonstrates tracer retention after $2.5 \mathrm{~h}$. (B) SPECT/CT confirming localization of adenoma (arrow) posterior to left lobe of thyroid. (C) Ultrasound showing tracer uptake corresponding to single hypoechoic nodule (arrow) posterior to left thyroid lobe in midgland region. Parathyroid adenomas have characteristic feeding artery that can often be seen on Doppler examination (not shown on this image).

\section{Statistical Analysis}

Preoperative imaging results were considered to be truepositives if they correctly identified the number of diseased glands and localized them to the correct side (right, left, or bilateral). To establish true positivity, radiologic reports were compared with the subsequent surgical pathology results. Like Patel et al. (6) and 
TABLE 1

Number of Diseased Glands by Frequency

\begin{tabular}{lc}
\hline Number of diseased glands & Frequency \\
\hline Single & $128(88 \%)$ \\
Multigland $(\geq 2)$ & $10(7 \%)$ \\
Negative exploration & $8(5 \%)$ \\
Total & 146 \\
\hline
\end{tabular}

Witteveen et al. (7), we considered nonidentification of normal glands on the unaffected side to represent a true-negative result. False-positives were defined as abnormal glands suggested on imaging in a location where none were found at surgery.

Secondarily, localization was also considered in terms of quadrants, as defined by Hinson et al. (8), where the patient's midline served to divide the gland into left and right and a line through the midthyroid served to divide superior from inferior. True-positives were defined as abnormal glands localized to correct quadrants, and true-negatives as the nonidentification of normal glands in the remaining quadrants.

The sensitivity, specificity, negative predictive value, and positive predictive value of the localization techniques were obtained using MedCalc statistical software (version 16.2.1; MedCalc Software). The sensitivities of SPECT/CT plus ultrasound and SPECT/CT alone were compared using the McNemar test; a $P$ value of 0.05 or less was taken to indicate statistical significance.

The sensitivity and specificity of combined SPECT/CT and ultrasound in patients with a normal thyroid gland were compared with those with Hashimoto thyroiditis, multinodular goitre, atrophic thyroid gland, or benign nodules using a Fisher exact test; a $P$ value of 0.05 or less was taken to indicate statistical significance.

SAS Studio (version 3.4; SAS Institute Inc.) was used to calculate the $P$ value with a $95 \%$ confidence interval.

The institutional review board approved this retrospective study and the requirement to obtain informed consent was waived in accordance with National Health Service Health Research Authority guidelines.

\section{RESULTS}

\section{Demographics}

Pathologic and radiologic data were available for a total of 148 patients who had undergone parathyroidectomy for primary hyperparathyroidism during the specified period. SPECT/CT combined with ultrasound was performed in
146 cases and SPECT/CT alone in 2 cases; these 2 cases were therefore excluded from further analysis. Of the remaining 146 patients, 115 were female and 31 male (ratio of 3.7:1). The median age was $62 \mathrm{y}$ (range, 23-88 y).

On the basis of the surgical and pathologic data, single adenomata were most common, with multigland disease being uncommon (Table 1). No abnormal glands were found in 8 cases $(5 \%)$.

\section{Imaging Modalities}

Overall, SPECT/CT-guided ultrasound (SPECT/CT followed by ultrasound performed by the same radiologist) correctly identified the number and laterality of abnormal glands in 123 cases, yielding an overall sensitivity of $83 \%$ (Table 2). On analyzing the technique's sensitivity for correctly localizing abnormal glands to a particular quadrant, the sensitivity fell slightly $(77 \%)$ when compared with lateralization alone; however, specificity remained high at $97 \%$ (Table 2). Of note, in 11 cases, the quadrant could not be established from the patients' postoperative electronic records, so that they could not be correlated with preoperative imaging. Therefore, these cases were excluded, leaving a total of 135 cases to be analyzed.

Ultrasound scanning helped localize lesions not identified by SPECT/CT in 13 cases (9\%). These were subsequently proven to be true-positives at surgery. Additionally, in 1 case, ultrasound successfully resolved 2 adjacent adenomata initially thought to represent a single lesion on SPECT/CT. Therefore, the additional component of ultrasound improved overall sensitivity (sensitivity of $83 \%$ for SPECT/CT plus ultrasound vs. sensitivity of $75 \%$ for SPECT/CT alone; $P<$ 0.005 using McNemar test) (Table 2).

Ultrasound was unable to confirm the presence of a lesion in 18 cases $(12 \%)$ that were positive on SPECT/CT. Again, these were proven to be true-positives at surgery. In these cases, failure of localization was attributed to the deep or atypical locations of abnormal glands (Fig. 2) or to patient factors (such as inability to extend the neck). Examples of discrepant cases on SPECT/CT and ultrasound are shown in Figure 3.

\section{Single- and Multigland Disease}

The data revealed a reduced sensitivity for SPECT/CTguided ultrasound in determining the correct side and gland number in the setting of multigland disease $(70 \%$; confidence

TABLE 2

Sensitivity, Specificity, Negative Predictive Value, and Positive Predictive Value According to Analysis and Modality

\begin{tabular}{lcccc}
\hline \multicolumn{1}{c}{ Analysis } & Sensitivity & Specificity & Negative predictive value & Positive predictive value \\
\hline Laterality and gland number $(n=146)$ & & & & \\
SPECT/CT plus ultrasound & $83(75.5-88.3)$ & $96(91.1-98.4)$ & $84(77.5-89.3)$ & $95(90.2-98.3)$ \\
SPECT/CT* & $75(66.8-81.5)$ & $96(91.3-98.5)$ & $79(72.4-84.8)$ & $95(89.0-98.1)$ \\
Quadrant and gland number $(n=135)$ & & & $92(89.1-94.5)$ & $89(82.5-94.2)$ \\
SPECT/CT plus ultrasound & $77(68.9-83.4)$ & $97(94.5-98.3)$ & $90(81.9-94.3)$
\end{tabular}

*Analyzed by disregarding ultrasound component of examination.

Data are percentage followed by $95 \%$ confidence interval in parentheses. 


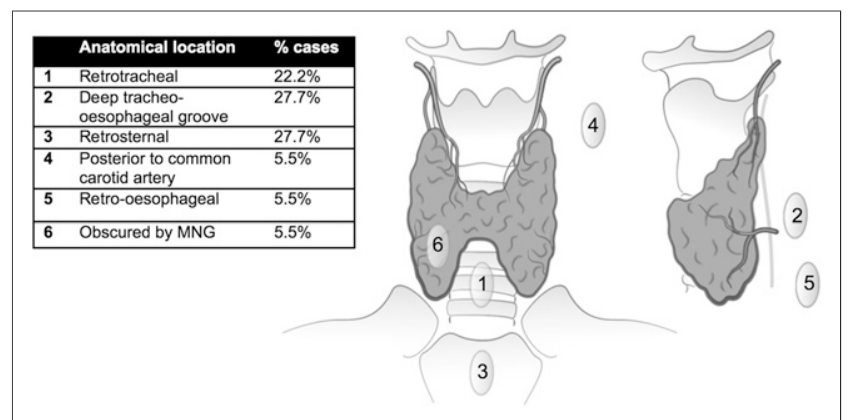

FIGURE 2. Adenomas not identified on ultrasound categorized by anatomic location. MNG = multinodular goiter.

interval, 34.8\%-93.3\%; $n=10)$, when compared with single-gland disease $(87 \%$, confidence interval, 79.6\%-92.1\%; $n=128)$.

\section{Coexisting Thyroid Disease}

The thyroid was reported as unremarkable or atrophic in 86 cases $(59 \%)$ and abnormal (nodular or ultrasonographic evidence of thyroiditis) in 60 cases (41\%). Among the latter, 4 patients $(7 \%)$ were recommended to undergo fineneedle aspiration as a result of their ultrasound examinations. The presence of concurrent thyroid disease did not adversely affect the sensitivity and specificity of SPECT/CT-guided ultrasound for lateralizing diseased glands (Table 3).

\section{DISCUSSION}

This study represents a large series reviewing the sensitivity and specificity of SPECT/CT-guided ultrasound in the preoperative localization of diseased parathyroid glands. The rationale of the technique is to offer patients the convenience of a single hospital attendance; it also offers the radiologist the opportunity to use SPECT/CT to guide the ultrasound examination. This opportunity enables as much anatomic information as possible to be provided to the operating surgeon, including the visibility of the adenoma for intraoperative ultrasound. Subsequently, a minimally invasive procedure can be offered in cases of clear single-gland disease, avoiding the risks associated with 4-gland exploration.
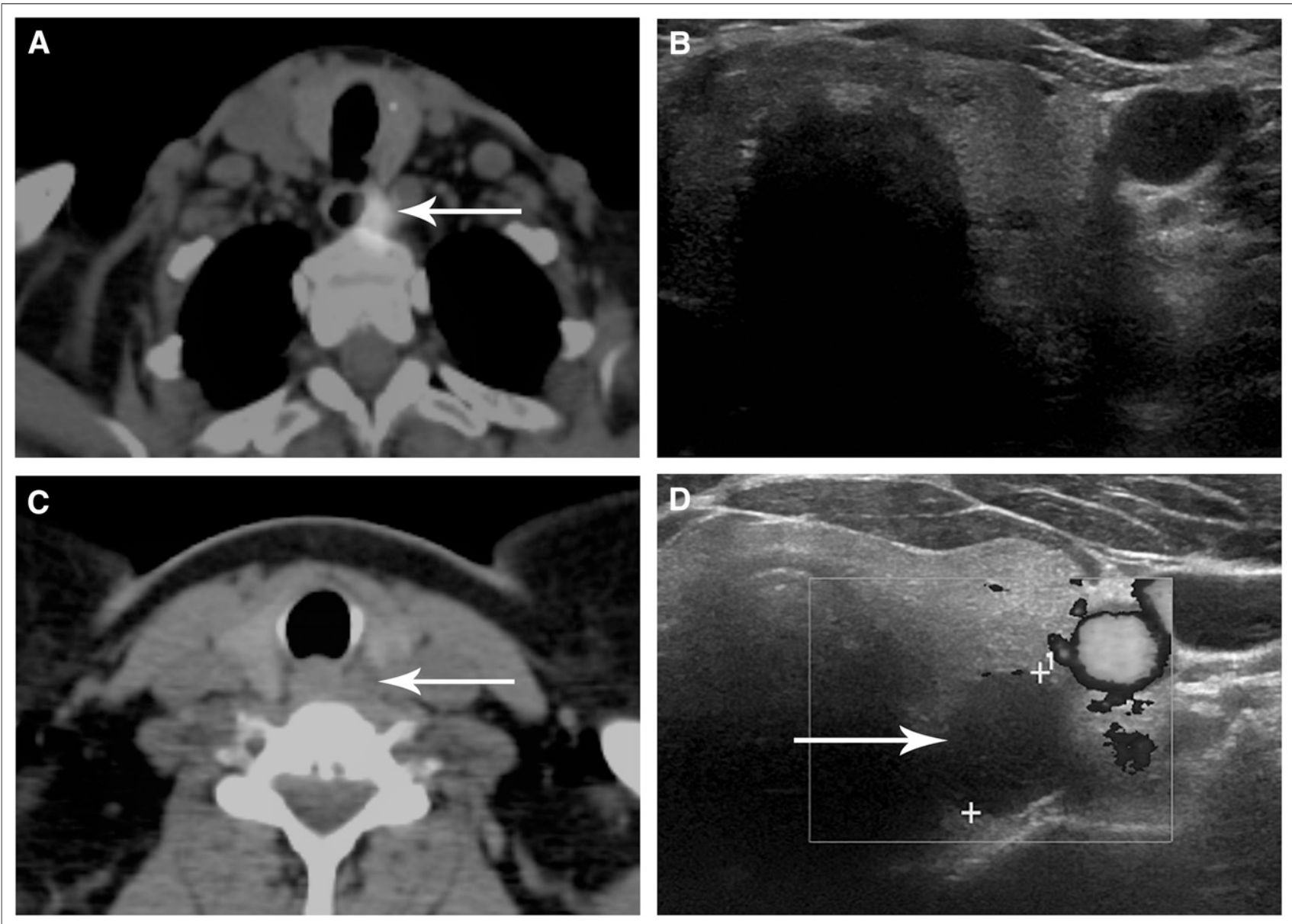

FIGURE 3. Series of images demonstrating discrepancies between modalities. (A) Avid tracer uptake is seen on SPECT/CT within left superior parathyroid adenoma (arrow), which is located deep in tracheoesophageal groove. (B) This finding is not visible on corresponding ultrasound because of acoustic shadowing from trachea but was subsequently located at surgery. (C) SPECT/CT reveals no tracer retention in nodule posterior to left lobe of thyroid (arrow). (D) On ultrasound, this nodule (arrow) appears to be characteristic of parathyroid adenoma, which has exhibited early washout with ${ }^{99 m}$ Tc-sestamibi. Diagnosis was confirmed at surgery. 
TABLE 3

Effect of Thyroid Disease on Localization of Parathyroid Adenomas with Combined SPECT/CT and Ultrasound

\begin{tabular}{lcccc}
\hline \multicolumn{1}{c}{ Finding } & Sensitivity & Specificity & Negative predictive value & Positive predictive value \\
\hline Normal $(n=86)$ & $82 \%$ & $97 \%$ & $85 \%$ & $96 \%$ \\
Atrophic $(n=5)$ & $100 \%(0.383)$ & $100 \%(0.847)$ & $100 \%$ & $100 \%$ \\
Benign nodules & $76 \%(0.194)$ & $100 \%(0.556)$ & $79 \%$ & $100 \%$ \\
$\quad$ (no glandular enlargement) $(n=20)$ & & & $100 \%$ \\
Hashimoto thyroiditis $(n=4)$ & $100 \%(0.462)$ & $100 \%(0.875)$ & $100 \%$ & $97 \%$ \\
Multinodular goiter $(n=60)$ & $94 \%(0.069)$ & $96 \%(0.429)$ & $93 \%$ & \\
\end{tabular}

Data in parentheses are probability values.

Our results yielded an overall sensitivity of $83 \%$ for all abnormal glands and $87 \%$ for single adenomata; the specificity was $96 \%$. These results are comparable with the literature $(9-11)$ but are slightly lower than found in some studies, which quote sensitivities of up to $96 \%(6,12,13)$. The reason for this difference is uncertain but could reflect the limitations of retrospective data acquisition. Indeed, our institution treats a large population of patients with tertiary hyperparathyroidism (which is associated with a lower lesion detection rate than primary hyperparathyroidism), and although every effort was made to exclude such cases, it is possible that some were included because of errors in the clinical documents used to identify patients. It is also possible that the single-phase SPECT/CT acquisition technique used in this study could have contributed to a slightly lower sensitivity.

Precise definition of gland localization is important, particularly as misclassification of superior and inferior parathyroid glands may be unhelpful to the surgeon, given the differing relationship to the recurrent laryngeal nerve. In this study, sensitivity was also calculated using the quadrant method suggested by Hinson et al. (8); its lower value (77\%) reflects the difficulties of applying a system of localization retrospectively and does not reflect the true sensitivity of the technique, as a superior parathyroid adenoma may lie deep to the inferior pole of the thyroid, leading to a discrepancy between the radiologic and surgical interpretation. A classification proposed by Perrier et al. seeks to provide more reliable nomenclature. It involves identifying multiple discrete cervical regions $(\mathrm{A}-\mathrm{G})$ that correspond to typical parathyroid locations (14). It has the potential benefit of improving communication of gland location between radiologists and surgeons but is yet to be widely used in the U.K.

As observed in other studies, there was a higher diagnostic sensitivity for localizing single-gland disease $(87 \%)$ than multigland disease $(70 \%)$. The reduced sensitivity was thought to be related to reduced gland weight and oxyphil content in hyperplastic glands (15-17). However, more recent studies have found no correlation between lesion weight and the reduced sensitivity of 99mTc-sestamibi SPECT/CT studies in multigland disease $(18,19)$.
This study highlights the utility of multimodality imaging. In our study, the additional use of ultrasound identified 13 cases (9\%), which were occult on SPECT/CT, resulting in a significant increase in sensitivity. The cause of nondetection by scintigraphy may relate to poor uptake by the lesion or early washout. A study by Lavely et al. (20) found a significant improvement in sensitivity if both early and delayed SPECT/CT acquisitions were obtained, as opposed to the early planar and delayed-phase SPECT/CT acquisition technique used in our institution. It is therefore possible that the sensitivity could have been increased further using this method.

The added value of multimodality imaging in adenoma detection is supported in the literature. However, there has been a suggestion that a single modality, such as ultrasound, could be used in the first instance, with the second modality used only as an adjunct when the ultrasound results are equivocal or negative $(21,22)$. Conversely, our data suggest that SPECT/CT could be used as the initial technique, with ultrasound used when an adenoma is not identified, as a slightly higher proportion of adenomata were occult on ultrasound than on SPECT/CT. However, ultrasound offers several additional benefits to support its routine use with SPECT/CT. In addition to its low cost, lack of ionizing radiation, and rapidity, its high spatial resolution enables resolution of adjacent adenomata, which may appear to represent a single gland on SPECT/CT. It also enables identification and characterization of thyroid disease that may mimic an adenoma on scintigraphy or require concurrent management.

In our experience, the ultrasound examination adds greater confidence in localizing adenomas that are visualized on SPECT/ CT. Anecdotally, there are cases in which the SPECT/CT findings are equivocal because of possible early washout of ${ }^{99 m}$ Tc-sestamibi, and the ultrasound scan allows interrogation of these specific areas. In our study, the radiologist was not masked to the results of the SPECT/CT when performing the ultrasound, so it was not possible to quantify this.

Finally, ultrasound is also helpful to surgeons performing subsequent intraoperative ultrasound and may add detail in specifying anatomic location. If there are discordant imaging findings between SPECT/CT and ultrasound, then intraoperative parathyroid hormone monitoring is proven to improve localization of adenomas $(23,24)$. 
Overall, the presence of preexisting thyroid disease does not seem to impair the detection of parathyroid adenomas, with no deleterious effect on the diagnostic sensitivity of SPECT/CTguided ultrasound in the presence of thyroid lesions. This finding is likely attributable to the use of combined modalities, as ultrasound can be of assistance in differentiating intrathyroidal lesions, which have uptake on SPECT, and scintigraphy is useful when lesions are obscured on ultrasound by large multinodular goiters. Ultrasound may also be used to evaluate incidental thyroid disease; in our series, 4 patients were referred for fineneedle aspiration as a result of their ultrasound examinations.

Because of the retrospective nature of the study, we were reliant on the quality of documentation and, as highlighted above, there can be discrepancies between the radiologic and surgical nomenclature of glands.

It was also not possible to compare the different modalities in isolation (ultrasound alone vs. SPECT/CT) because a single combined protocol was used. The radiologist was not masked to the results of the SPECT/CT when performing the ultrasound examination. In fact, this characteristic was felt to be one of the strengths of the technique because the SPECT/CT could be used to target specific areas with ultrasound to improve confidence in reporting findings on SPECT/CT and ultrasound combined. However, this characteristic could bias the results of the SPECT/CT.

Additionally, because the focus was on patients undergoing surgery, the study did not identify those with positive scans who did not undergo surgery (e.g., because they chose not to or had other comorbidities).

\section{CONCLUSION}

SPECT/CT-guided ultrasound offers a reliable means of localizing parathyroid adenomata in primary hyperparathyroidism, and the 1-stop approach offers patient convenience and aids diagnostic confidence. The modalities in combination demonstrate a significant increase in sensitivity. Furthermore, concurrent thyroid disease does not impair localization of parathyroid adenomas. However, preoperative localization is more accurate with single adenomas than with multigland disease, as correlates with the published literature.

\section{DISCLOSURE}

No potential conflict of interest relevant to this article was reported.

\section{ACKNOWLEDGMENTS}

We thank all staff at the Department of Radiology at St. Helier Hospital and our colleagues in the Department of Pathology and Head and Neck Surgery. Preliminary data were presented at the European Congress of Radiology (ECR) in March 2016.

\section{REFERENCES}

1. Felger EA, Kandil E. Primary hyperparathyroidism. Otolaryngol Clin North Am. 2010;43:417-432.
2. Adler JT, Sippel RS, Chen H. The influence of surgical approach on quality of life after parathyroid surgery. Ann Surg Oncol. 2008;15:1559-1565.

3. Chen H, Sokoll LJ, Udelsman R. Outpatient minimally invasive parathyroidectomy: a combination of sestamibi-SPECT localization, cervical block anesthesia, and intraoperative parathyroid hormone assay. Surgery. 1999;126:10161021 .

4. Grubbs EG, Edeiken BS, Gule MK, Monroe BJ, Vu EKT, Perrier ND. Preoperative parathyroid imaging for the endocrine surgeon. In: Handbook of Parathyroid Diseases: A Case-Based Practical Guide. New York, NY: Springer; 2012: 19-40.

5. Randolph GW. Applied embryology of the thyroid and parathyroid glands. In: Surgery of the Thyroid and Parathyroid Glands. 2nd ed. Philadelphia, PA: Elsevier Health Sciences; 2012;15.

6. Patel CN, Salahudeen HM, Lansdown M, Scarsbrook AF. Clinical utility of ultrasound and ${ }^{99 \mathrm{~m}} \mathrm{Tc}$ sestamibi SPECT/CT for preoperative localization of parathyroid adenoma in patients with primary hyperparathyroidism. Clin Radiol. 2010;65:278-287.

7. Witteveen JE, Kievit J, Stokkel MP, Morreau H, Romijn JA, Hamdy NA. Limitations of Tc99m-MIBI-SPECT imaging scans in persistent primary hyperparathyroidism. World J Surg. 2011;35:128-139.

8. Hinson AM, Lee DR, Hobbs BA, Fitzgerald RT, Bodenner DL, Stack BC Jr. Preoperative 4D CT localization of nonlocalizing parathyroid adenomas by ultrasound and SPECT-CT. Otolaryngol Head Neck Surg. 2015;153:775-778.

9. Cheung K, Wang TS, Farrokhyar F, Roman SA, Sosa JA. A meta-analysis of preoperative localization techniques for patients with primary hyperparathyroidism. Ann Surg Oncol. 2012;19:577-583.

10. Ruda JM, Hollenbeak CS, Stack BC Jr. A systematic review of the diagnosis and treatment of primary hyperparathyroidism from 1995 to 2003. Otolaryngol Head Neck Surg. 2005;132:359-372.

11. Wong KK, Fig LM, Gross MD, Dwamena BA. Parathyroid adenoma localization with ${ }^{99 \mathrm{~m} T c-s e s t a m i b i}$ SPECT/CT: a meta-analysis. Nucl Med Commun. 2015;36: 363-375.

12. De Feo ML, Colagrande S, Biagini C, et al. Parathyroid glands: combination of ${ }^{99 \mathrm{~m}} \mathrm{Tc}$ MIBI scintigraphy and US for demonstration of parathyroid glands and nodules. Radiology. 2000;214:393-402.

13. Lumachi F, Zucchetta P, Marzola MC, et al. Advantages of combined technetium-99m-sestamibi scintigraphy and high-resolution ultrasonography in parathyroid localization: comparative study in 91 patients with primary hyperparathyroidism. Eur J Endocrinol. 2000;143:755-760.

14. Perrier ND, Edeiken B, Nunez R, et al. A novel nomenclature to classify parathyroid adenomas. World J Surg. 2009;33:412-416.

15. Sepahdari AR, Bahl M, Harari A, Kim HJ, Yeh MW, Hoang JK. Predictors of multigland disease in primary hyperparathyroidism: a scoring system with 4DCT imaging and biochemical markers. AJNR Am J Neuroradiol. 2015;36:987992.

16. Elgazzar AH, Alenezi SA. Parathyroid gland. In: Elgazzar AH, ed. The Pathophysiologic Basis of Nuclear Medicine. New York, NY: Springer; 2014:222237.

17. Mehta NY, Ruda JM, Kapadia S, Boyer PJ, Hollenbeak CS, Stack BC Jr. Relationship of technetium Tc 99m sestamibi scans to histopathological features of hyperfunctioning parathyroid tissue. Arch Otolaryngol Head Neck Surg. 2005;131: 493-498.

18. Nichols KJ, Tomas MB, Tronco GG, Palestro CJ. Sestamibi parathyroid scintigraphy in multigland disease. Nucl Med Commun. 2012;33:43-50.

19. Nichols KJ, Tronco GG, Palestro CJ. Influence of multigland parathyroid disease on ${ }^{99 \mathrm{~m}}$ Tc-sestamibi SPECT/CT. Clin Nucl Med. 2016;41:282-288.

20. Lavely WC, Goetze S, Friedman KP, et al. Comparison of SPECT/CT, SPECT, and planar imaging with single- and dual-phase ${ }^{99 \mathrm{~m}} \mathrm{Tc}$-sestamibi parathyroid scintigraphy. J Nucl Med. 2007;48:1084-1089.

21. Tublin ME, Pryma DA, Yim JH, et al. Localization of parathyroid adenomas by sonography and technetium Tc 99m sestamibi single-photon emission computed tomography before minimally invasive parathyroidectomy: are both studies really needed? J Ultrasound Med. 2009;28:183-190.

22. Untch BR, Adam MA, Scheri RP, et al. Surgeon-performed ultrasound is superior to ${ }^{99} \mathrm{Tc}$-sestamibi scanning to localize parathyroid adenomas in patients with primary hyperparathyroidism: results in 516 patients over 10 years. J Am Coll Surg. 2011;212:522-529.

23. Calò PG, Pisano G, Loi G, et al. Surgery for primary hyperparathyroidism in patients with preoperatively negative sestamibi scan and discordant imaging studies: the usefulness of intraoperative parathyroid hormone monitoring. Clin Med Insights Endocrinol Diabetes. 2013;6:63-67.

24. Lew JI, Solorzano CC, Montano RE, Carneiro-Pla DM, Irvin GL III. Role of intraoperative parathormone monitoring during parathyroidectomy in patients with discordant localization studies. Surgery. 2008;144:299-306. 